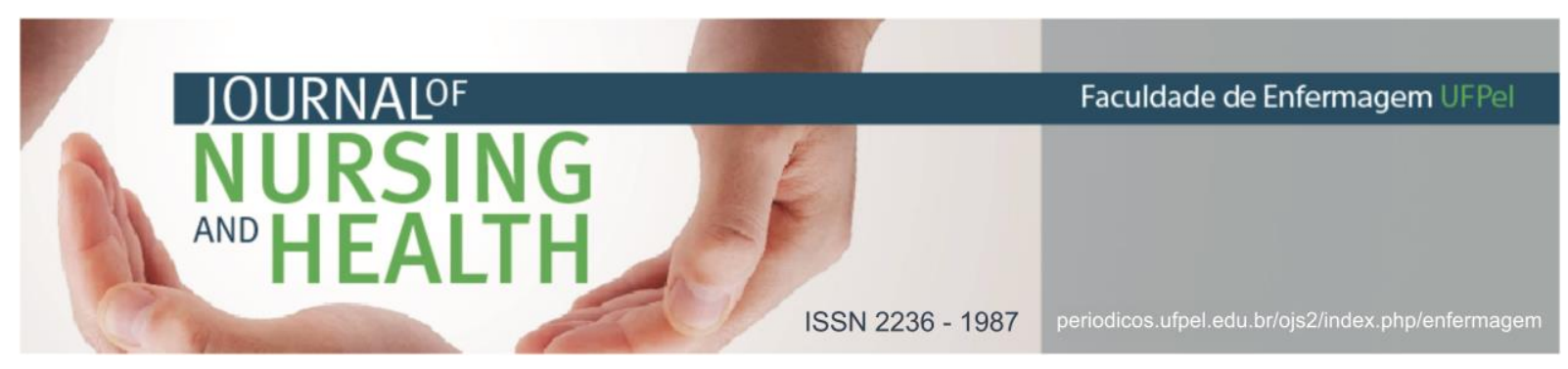

ARTIGO DE REVISÃO

\title{
Benefícios de acompanhante treinada durante o processo de parturição: revisão integrativa
}

\author{
The benefits of an attendant trained during the parturition process: integrative \\ review
}

\section{Las ventajas de un compañero capacitado durante el proceso del parto: una revisión integrativa}

Almeida, Keurolainy Cristine Silva ${ }^{1}$; Siqueira, Hedi Crecencia Heckler de ${ }^{2}$; Pereira, Queli Lisiane Castro $^{3}$

\begin{abstract}
RESUMO
Objetivo: conhecer a produção científica acerca dos benefícios de acompanhante treinada durante o processo de parturição. Métodos: revisão integrativa. Na busca ativa online nas bases de dados, utilizou-se os descritores, parto humanizado, assistência ao paciente e trabalho de parto. Observouse como critérios de inclusão, artigos publicados na língua portuguesa, disponíveis online, completos, publicados no período de 2001 a 2015. A coleta de dados foi realizada em janeiro de 2016. Resultados: foram capturados 11 artigos. Os dados evidenciaram que a participação da acompanhante treinada no processo de parturição traz como benefícios: a humanização do parto, a realização de medidas de alívio da dor, a redução de intervenções e o aumento de partos eutócicos, além de causar satisfação e vivência positiva das parturientes acompanhadas por doulas. Considerações finais: novos estudos devem ser realizados a fim de impulsionar a presença das doulas nas maternidades.

Descritores: Parto humanizado; Assistência ao paciente; Trabalho de parto.
\end{abstract}

\begin{abstract}
Objective: to know the scientific production about the accompanying benefits trained during the parturition process. Methods: integrative review. In online active search in databases, descriptors were used: humanized delivery, patient care, and labor. It was observed as inclusion criteria articles published in Portuguese, available online, full-text, from 2001 to 2015. Data collection was conducted in January 2016. Results: 11 articles were captured. The data showed that the participation of accompanying trained in the parturition process brings the following benefits: birth humanization, the realization of pain relief measures, the reduction interventions and increased eutocic births and cause positive satisfaction and experience of mothers accompanied by doulas. Final considerations: further studies should be conducted in order to boost the presence of doulas in maternity wards.
\end{abstract}

Descriptors: Humanizing delivery; Patient care; Labor, obstetric.

\footnotetext{
1 Enfermeira. Docente do curso Técnico em Enfermagem do SENAC. Barra do Garças, MT, Brasil. E-mail: kero2015ufmt@outlook.com

2 Enfermeira. Doutora em Enfermagem. Professora emérita, titular da Fundação Universidade Federal do Rio Grande no curso de pós-graduação - Mestrado e Doutorado. Líder do Grupo de Estudo e Pesquisa Gerenciamento Ecossistêmico em Enfermagem/Saúde (GEES). Rio Grande, RS, Brasil. E-mail: hedihsiqueira@gmail.com

${ }^{3}$ Enfermeira. Doutora. Professora Adjunta do Curso de Bacharelado em Enfermagem do Campus Universitário do Araguaia (CUA) da Universidade Federal de Mato Grosso (UFMT). Membro do grupo de estudo e pesquisa GEES. Barra do Garças, Mato Grosso, Brasil. E-mail: quelilisiane@hotmail.com
} 


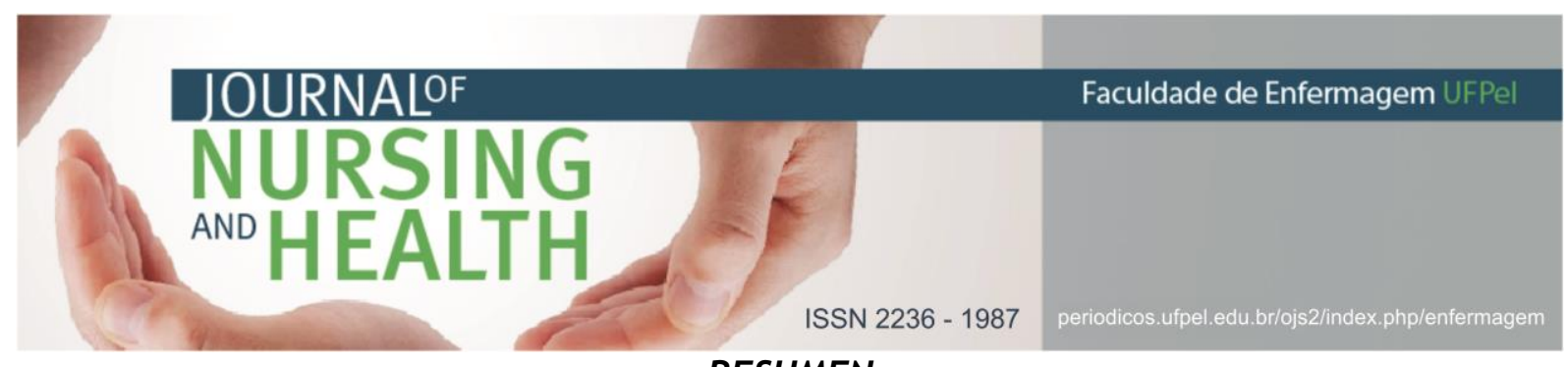

RESUMEN

Objetivo: conocer la producción científica sobre los beneficios que se acompañan formados durante el proceso de parto. Métodos: revisión integradora. En la búsqueda activa en línea en bases de datos, se utilizan descriptores, parto humanizado, atención al paciente y trabajo de parto. Se observó que los criterios de inclusión artículos publicados en portugués, disponible en línea, completa, publicados entre 2001 y 2015. La recolección de datos se llevó a cabo en enero de 2016. Resultados: 11 artículos fueron capturados. Los datos mostraron que la participación de acompañar entrenado en el proceso del parto trae los siguientes beneficios: humanización del nacimiento, la realización de medidas de alivio del dolor, las intervenciones de reducción y el aumento de los partos eutócicos y causan satisfacción y experiencia positiva de madres acompañadas por doulas. Consideraciones finales: más estudios deben llevarse a cabo con el fin de impulsar la presencia de doulas en las maternidades.

Descriptores: Parto humanizado; Atención al paciente; Trabajo de parto.

\section{INTRODUÇÃO}

O processo caracterizado pelo parto e nascimento, no século XIX, era assistido por familiares, comumente mulheres, até a sua institucionalização. A partir de então, o atendimento concentrou-se no âmbito hospitalar, realizado pelos profissionais de saúde, assim, passou a envolver uma série de riscos, gerando experiências insatisfatórias à mulher. Algumas práticas que eram incentivadas têm tido resultados benéficos para as parturientes, principalmente, por meio do apoio de doulas que as preparam tanto emocionalmente, quanto fisicamente para o processo de parturiçãa. ${ }^{1}$

De acordo com a Organização Mundial de Saúde (OMS), a doula presta serviço à mulher durante o ciclo gravídico, tendo treinamento básico e familiarização com os procedimentos de assistência. ${ }^{2}$ Para o Ministério da Saúde (MS), a doula é uma acompanhante treinada que oferece apoio emocional, informações à parturiente, bem como, executa intervenções e procedimentos necessários para que a mulher possa participar de fato das decisões acerca das condutas a serem tomadas durante este período. ${ }^{3}$

Há certa ambiguidade quanto ao papel e status da doula no acompanhamento das parturientes. São consideradas doulas, mulheres voluntárias ou pagas para oferecer o suporte, com ou sem experiência técnica na área da saúde, que desempenham atividades estritamente relacionadas às necessidades de conforto físico/emocional da parturiente, ou que também atuam na transmissão de instruções médicas. ${ }^{4}$

A formação ainda não é regulamentada, assim, ela ocorre de forma variável, presencial, fundamentada nas orientações da OMS e com carga horária diversa. Em relação a carga horária prática, essa ocorre, em alguns casos, somente a nível de laboratório, enquanto em outros, é exigido doular duas parturientes durante o período de treinamento. ${ }^{4}$ No Hospital Sofia Feldman de Belo Horizonte, para implementar o Projeto Doulas "Ajudando a nascer, o treinamento às mulheres sem experiência na área da saúde foi concluído em $40 \mathrm{~h} .{ }^{5}$ Já o curso de extensão para formação de doulas 


\section{JOURNALOF \\ NURSING \\ AND HEALTH}

ISSN 2236 - 1987

do Instituto Federal de Brasília (IFDF) possui carga horária de 205 horas. ${ }^{6}$ Há diversidade quanto à formação e nível de treinamento, das doulas, embora seja uma ocupação regulamentada pela Classificação Brasileira de Ocupações (CBO), código 3221-35, qualificada como uma ocupação da área da saúde.

Nos Estados Unidos, em 1976, em decorrência do aumento do número de cesarianas e das sucessivas buscas por apoio para reduzir os índices cirúrgicos, houve o reconhecimento da doula, que se tornou popular devido à necessidade de auxílio às mulheres, oferecendo instruções no trabalho de parto eutócico. De acordo com a Associação de Doulas da América do Norte (DONA), a doula é uma mulher capacitada a prestar apoio emocional, físico e assistência à mulher no trabalho de parto. A palavra doula é de origem grega e significa escrava. Antigamente davam assistência em casa após o parto, atualmente desempenha uma função que antecede o parto, desde a preparação até a amamentação. ${ }^{7}$

Ainda que a Doula seja conhecida em outros países desde a década de 1970 , só atualmente vem sendo conhecida no Brasil e, aos poucos, vem conquistando seu espaço nos grandes centros, já que são evidentes os benefícios de se ter uma acompanhante treinada durante a gestação, parto e pós-parto. Estudos científicos evidenciaram que as gestantes que tiveram a presença de acompanhantes, sentiram-se mais seguras e confiantes durante 0 parto. ${ }^{1,4-5,7}$ Além disso, reduziram o uso de medicações para alívio da dor, da duração do trabalho de parto e do número de cesáreas. Outro estudo, sugere, inclusive, a possibilidade de redução dos casos de depressão pósparto. ${ }^{8}$

São atribuições da Doula, segundo - MS: Auxiliar a mulher a encontrar uma posição confortável durante as contrações; proporcionar um ambiente calmo, silencioso, acolhedor para realizar as técnicas respiratórias; oferecer medidas de conforto por meio de massagens, de banhos mornos; realizar técnicas para auxiliar no alívio/controle da dor. Além disso, cabe à Doula promover a interação do marido ou companheiro durante todo o período; esclarecer as dúvidas da mulher durante o período expulsivo e dar liberdade de escolha da posição. ${ }^{3}$

Da mesma forma compete à Doula, oferecer à parturiente apoio emocional, e proporcionar a comunicação, entre os profissionais e os familiares, almejada pela mulher. $\mathrm{E}$, em relação à puérpera, carece de incentivar a promoção da amamentação e o vínculo dos pais com o recém-nascido ( $R N)$; realizar visita domiciliar para acompanhar 0 desenvolvimento do $\mathrm{RN}$ e verificar a necessidade de orientações à puérpera. ${ }^{1}$

Conforme estabelece o Programa de Humanização no Pré-natal e Nascimento, tornou-se um direito de toda a gestante, um atendimento humanizado, digno e com condições adequadas, assegurando que tenha acesso integral durante a gestação, pré-natal, parto e assistência assistida à puérpera e ao recém-nascido. ${ }^{3}$ Assim sendo, o processo de humanização, no atendimento e assistência prestada às 
gestantes no período puerperal, envolve alguns aspectos inovadores e desafiadores, tanto no serviço prestado quanto na reformulação de um novo modelo de atendimento humanizado. ${ }^{8-10}$

0 enfrentamento do modelo de atenção ao parto excessivamente intervencionista desenvolvido no país foi questionado no Programa de Humanização do Pré-natal e Nascimento (PHPN), criado pelo MS no ano 2000. Esse programa apresenta, como um de seus fundamentos, o direito à humanização da assistência obstétrica e neonatal. Entre seus princípios, instituiu o acompanhamento adequado à mulher no ciclo gravídico-puerperal e recémnascidos, estabelece o incentivo ao parto natural e presença de um acompanhante escolhido pela parturiente, para que esse momento se torne o mais confortável possível. ${ }^{10}$ Portanto, esse programa representa um contraponto ao modelo intervencionista do parto. ${ }^{11}$

Em relação à presença da doula no trabalho de pré-parto e parto, traz benefícios tanto para a parturiente quanto para o RN, aplicando um modelo humanizado de atendimento, que evidencia melhor desempenho de cuidados e vínculo da mãe com o RN, contribuindo também para a redução do número de anestesia peridural para parto vaginal, diminuição do uso de ocitocina, menor taxa de cesarianas, diminuição do tempo de trabalho de parto, menor índice de RN com sepses neonatal e de depressão puerperal relacionado à elevada média de apoio e autoconfiança e à diminuição de ansiedade. ${ }^{1,9}$
$\mathrm{Na}$ mesma linha de pensamento, os métodos não farmacológicos vêm sendo incentivados pelo MS por meio de práticas e métodos capazes de interferir no alívio da dor durante o trabalho de parto, como técnicas de respiração, massagem, deambulação e mudança de posição e a presença e o suporte da doula. ${ }^{12}$

Nessa acepção, o processo de acompanhamento à parturiente envolve ainda os benefícios do uso de técnicas de relaxamento por meio da biomúsica, que interfere no processo respiratório tornando-o mais lento e profundo, aumenta a aptidão e excitações sensoriais, elenca o domínio afetivo e uma boa regulação fisiológica. ${ }^{13}$ Os princípios do cuidar durante o processo de fragilidade da vida da mulher, não implicam apenas em aliviar a dor. É de fundamental importância escutar, dar conforto físico e emocional, apoio moral e psicológico durante todo o processo de parturição, informando sobre o que está sendo, ou será feito, tornando, desta forma, esse momento de vida da mulher uma experiência satisfatória. ${ }^{14}$

Torna-se evidente que a influência nos resultados obtidos se dá pela atribuição da doula ao lado da parturiente, tranquilizando e encorajando, interferindo, assiduamente, nos níveis de catecolaminas (adrenalina e noradrenalina) que são liberadas na circulação em situações de ansiedade intensa. Desta forma, o trabalho da doula tem uma função importante no suporte intraparto. Isto posto, torna-se necessário acompanhar o processo de implantação da humanização do atendimento prestado pela doula 


\section{JOURNALOF \\ NURSING \\ M०HEALTH}

ISSN 2236 - 198

conhecimento produzido sobre o tema parto acompanhado por doula.

Para elaboração da presente revisão integrativa, foram percorridas seis etapas. ${ }^{15}$

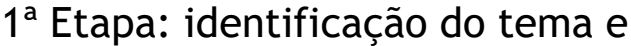
seleção da questão de pesquisa, ou seja, conhecer os benefícios de uma acompanhante treinada durante 0 processo de parturição. Vale enfatizar, que a base fundamental para elaboração da questão norteadora foi a revisão de literatura.

$2^{\text {a }}$ Etapa: estabelecimento de critérios de inclusão e exclusão.

0 estudo foi realizado por meio de busca online das produções científicas nacionais sobre o parto acompanhado por Doula, no período de 2001 a 2015. A captura dessas produções foi processada por meio da Biblioteca Virtual em Saúde (BVS), sendo utilizadas as bases de dados: Literatura Latino-Americana e do Caribe em Ciências da Saúde (LILACS) e Scientific Electronic Library Online (SciELO).

Estabeleceram-se como critérios para a seleção da amostra: artigos publicados, no período de 2001 a 2015, estar disponível integralmente e apresentar de maneira explícita os descritores parto humanizado, assistência ao paciente em trabalho de parto e trabalho de parto no resumo. $\mathrm{Na}$ busca, utilizou-se os descritores em Ciências da Saúde (DeCS): "parto humanizado", "assistência ao paciente" e "trabalho de parto". Limitou-se a esses, pois, ao associar um quarto descritor, os artigos eram escassos, impossibilitando uma pesquisa aprofundada. Utilizou-se 0 


\section{NURSING AND HEALTH}

\section{ISSN 2236 - 1987}

operador booleano OR para que pelo menos um dos termos digitados fosse encontrado.

Para exclusão, tomou-se cuidado com os artigos que se repetiam nas bases de dados. A partir disso foram encontrados 19 artigos, dos quais 11 foram selecionados sendo; quatro indexados na LILACS e sete na SciELO e três excluídos por repetição nas bases de dados (Figura 1)

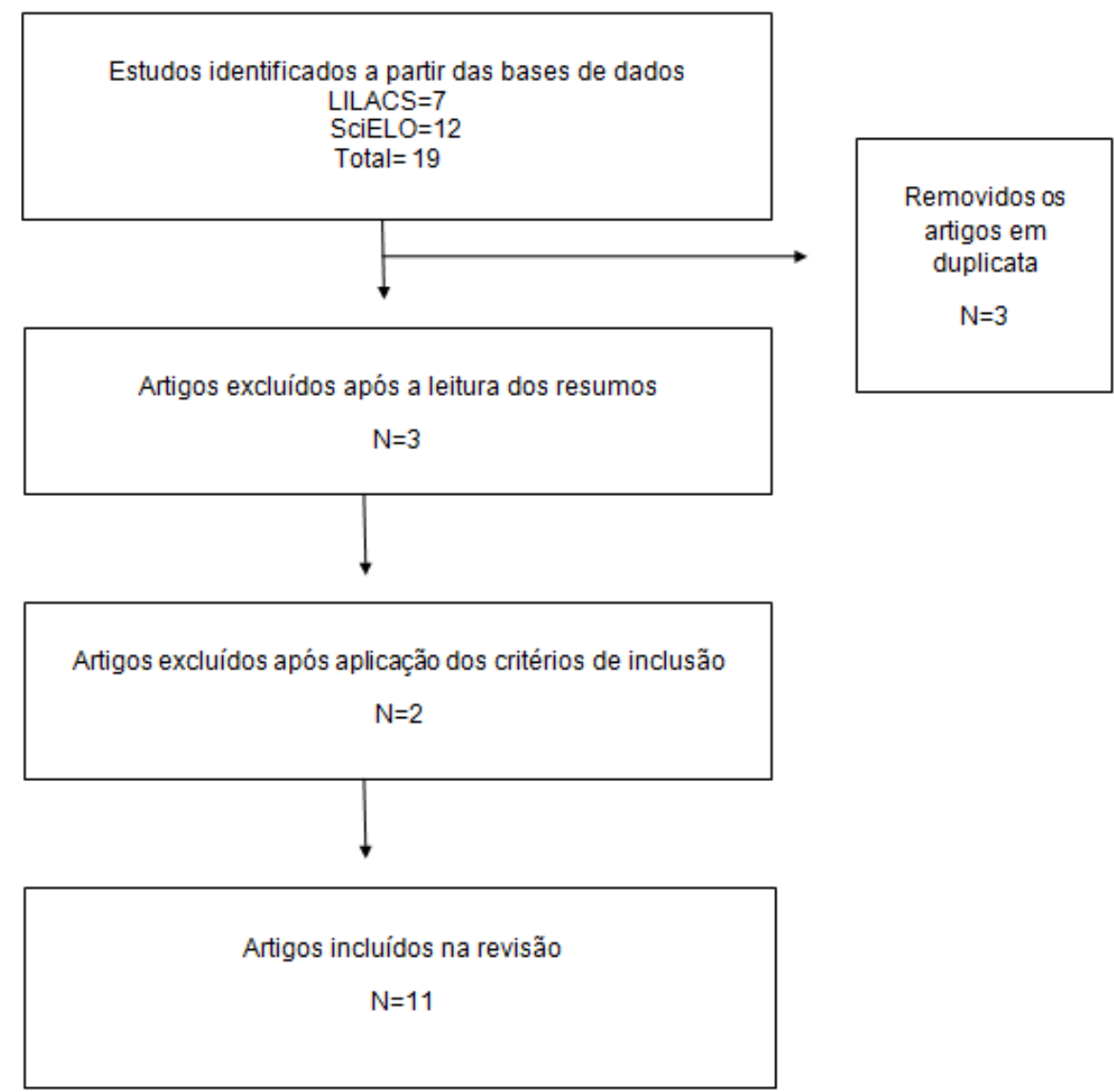

Figura 1: Fluxograma de identificação e seleção dos artigos para Revisão Integrativa.

$3^{\mathrm{a}}$ Etapa: Identificacão dos previamente estabelecidos e a estudos pré-selecionados e selecionados.

A partir das publicações completas localizadas pela estratégia de busca, procedeu-se, por inúmeras vezes, a leitura do material, a fim de evidenciar e delimitar o que se faz indispensável para a obtenção de um estudo aprofundado. Para isso, considerou-se a temática apresentada no seu enquadramento dos critérios aderência ao objetivo proposto.

A partir da referida identificação dos artigos e enquadramento aos critérios determinados na segunda etapa do método, obteve-se 11 artigos (Figura 1).

$4^{\mathrm{a}}$ Etapa: Categorização dos estudos selecionados.

$\mathrm{Na}$ quarta etapa, buscou-se sumarizar e documentar as informações extraídas dos artigos 
científicos encontrados nas fases anteriores. Para extrair os dados dos artigos selecionados, fez-se necessária a utilização de um instrumento previamente elaborado a fim de assegurar que a totalidade dos dados relevantes fosse extraída, minimizar o risco de erros na transcrição, garantir precisão na checagem das informações e servir como registro. Utilizou-se o instrumento para coleta de dados validada ${ }^{16}$, de forma adaptada.

Foram incluídos, desta forma, os seguintes dados: definição dos sujeitos, metodologia, tamanho da amostra, mensuração de variáveis, método de análise e conceitos embasadores empregados.

Ao final desta etapa, foi possível extrair as informações dos artigos e o instrumento permitiu analisar separadamente cada artigo, tanto no nível metodológico quanto em relação aos resultados das pesquisas, além de possibilitar a síntese dos artigos e salvaguardar suas diferenças.

$5^{a}$ Etapa: Análise e interpretação dos resultados.

Análoga à análise dos dados das pesquisas convencionais, esta fase demandou uma abordagem organizada para ponderar 0 rigor $e$ as características de cada estudo. No seguimento, para facilitar a visualização estrutural e lógica do estudo, fez-se a confecção de fichas de leitura, com o intuito de destacar as unidades de registro para agrupar os diferentes dados.

$6^{\text {a }}$ Etapa: Apresentação da revisão/síntese do conhecimento.
Essa etapa contempla os principais resultados obtidos $\mathrm{e}$ as propostas para futuros estudos.

\section{RESULTADOS E DISCUSSÃO}

Constatou-se que em relação ao ano da publicação dos artigos, compreendidos entre 2001 e 2015, os dados coletados apresentaram a distribuição, a seguir, um (9\%) de 2001, dois (18\%), em 2007, um (9\%) de 2009, três $(27 \%)$ foram publicados em 2010 , dois $(18 \%)$ em de 2012 , dois (18\%) de 2014 (Quadro 1).

No que tange a graduação dos autores, nove (82\%) eram enfermeiros, sendo em apenas um (9\%) tinha enfermeiro e médico e um (9\%) médicos. No que se refere ao país de publicação, 100\% dos trabalhos são nacionais.

Quanto aos locais de realização das 11 pesquisas analisadas, estavam assim distribuídos: quatro (36\%) de São Paulo, duas (18\%) no Ceará, as demais foram realizadas no estado de Minas Gerais, Santa Catarina, Piauí, Bahia, Pernambuco, contribuindo com um artigo (9\%), respectivamente.

Em suma, dos onze trabalhos nacionais, no período de 2001 a 2015, identificou-se o maior número de publicações, três (27\%), no ano de 2010, a maioria dos autores, nove (82\%), eram enfermeiros e o estado de São Paulo foi o local no qual foram realizadas a maioria das pesquisas, quatro (36\%), no período supracitado.

$\mathrm{Na}$ classificação dos estudos, quanto ao contexto em que foram desenvolvidas as pesquisas, a maioria delas, seis (54\%), foram realizadas em instituições de saúde. Desses, dois 


\section{NURSING \\ AND HEALTH}

ISSN 2236 - 1987

(32\%) em maternidade, um (16\%) em unidade básica e maternidade, um (16\%) na unidade básica de saúde, um (16\%) em hospital universitário e um (16\%) no instituto de perinaltologia. Os outros estudos, cinco (45\%), foram desenvolvidos em instituições de ensino superior e as coletas foram realizadas online.

\begin{tabular}{|c|c|c|c|}
\hline $\begin{array}{c}\mathrm{N}^{\circ} \\
\text { Artigo }\end{array}$ & $\begin{array}{l}\text { Ano } \\
\text { Publ. }\end{array}$ & Título & Autores \\
\hline 1 & 2010 & $\begin{array}{l}\text { Participação do acompanhante na } \\
\text { humanização do parto e sua relação } \\
\text { com a equipe de saúde }\end{array}$ & $\begin{array}{l}\text { Longo CSM, Andraus LMS, Barbosa } \\
\text { MA. }\end{array}$ \\
\hline 2 & 2010 & $\begin{array}{l}\text { Efeito da música no trabalho de } \\
\text { parto e no recém-nascido }\end{array}$ & Tabarro CS, Campos LB de Galli NO. \\
\hline 3 & 2012 & $\begin{array}{l}\text { Evidências qualitativas sobre } 0 \\
\text { acompanhamento por doulas no } \\
\text { trabalho de parto e no parto }\end{array}$ & $\begin{array}{c}\text { Silva RM da, Barros NF de, Jorge } \\
\text { Herla MF, Melo LPT, Ferreira Junior } \\
\text { AR. }\end{array}$ \\
\hline 4 & 2014 & $\begin{array}{l}\text { Métodos não farmacológicos para o } \\
\text { alívio da dor no trabalho de parto: } \\
\text { revisão integrativa }\end{array}$ & Mafetoni RR, Shimo AKK. \\
\hline 5 & 2007 & $\begin{array}{l}\text { A vivência de mulheres no parto } \\
\text { domiciliar e hospitalar }\end{array}$ & Crizóstomo CD, Nery IS, Luz MHB. \\
\hline 6 & 2012 & $\begin{array}{l}\text { Atuação da enfermagem na } \\
\text { humanização do parto: uma revisão } \\
\text { integrativa }\end{array}$ & $\begin{array}{c}\text { Lima M da S, Moreira KA, Martins- } \\
\text { Melo FR. }\end{array}$ \\
\hline 7 & 2007 & $\begin{array}{l}\text { Cuidado e conforto durante o } \\
\text { trabalho de parto e parto: na busca } \\
\text { pela opinião das mulheres }\end{array}$ & $\begin{array}{l}\text { Carraro TE, Knobel R, Radünz Vera, } \\
\text { Meincke SMK, Fiewski MFC, Frello AT, } \\
\text { Martins M da S, Lopes CV, Berton A. }\end{array}$ \\
\hline 8 & 2001 & $\begin{array}{l}\text { Doulas apoiando mulheres durante o } \\
\text { trabalho de parto: experiência do } \\
\text { hospital Sofia Feldman }\end{array}$ & Leão MRC, Bastos MAR. \\
\hline 9 & 2009 & $\begin{array}{l}\text { Doulas na assistência ao parto: } \\
\text { concepção de profissionais de } \\
\text { enfermagem }\end{array}$ & Santos D da S, Nunes IM. \\
\hline 10 & 2010 & $\begin{array}{l}\text { História oral: a experiência das } \\
\text { doulas no cuidado à mulher }\end{array}$ & Souza KRF de, Dias MD. \\
\hline 11 & 2014 & $\begin{array}{l}\text { A bola Suíça no alívio da dor de } \\
\text { primigestas na fase ativa do trabalho } \\
\text { de parto }\end{array}$ & $\begin{array}{c}\text { Gallo RBS, Santana LS, Marcolin AC, } \\
\text { Quintana SM. }\end{array}$ \\
\hline
\end{tabular}

Quadro 1 - Estudos selecionados pela pesquisa: $\mathrm{n}^{\circ}$ do artigo, ano de publicação, título e autores

Fonte: Dados da pesquisa organizados pelas pesquisadoras

Ao analisar as metodologias utilizadas pelos autores, quatro (36\%) empregaram a Revisão integrativa, três $(27 \%)$ a abordagem qualitativa, um (9\%) quantitativa, um $\quad(9 \%)$ metassíntese, um qualiquantitativa e um (9\%) ensaio clinico randomizado.
Após a análise quantitativa dos dados, optou-se em utilizar o método de análise temática para analisar, interpretar e agrupar os dados semelhantes. Assim, emergiram duas categorias, vivência do acompanhamento por doula, dois $(18 \%)$, e práticas usadas no alívio da dor, 11 (100\%). 


\section{JOURNALOF \\ NURSING \\ ANO HEALTH \\ Vivência do acompanhamento por doula}

As pesquisas ${ }^{7,9}$ esboçaram a vivência das mulheres que tiveram o acompanhamento por doula. Nos referidos estudos, foi possível identificar vivências positivas no processo de parturição. As mulheres referiram maior segurança, confiança, terem ficado mais calmas e relaxadas frente à atenção recebida pelas doulas.

A mulher busca um ambiente em que se sinta cuidada, com oferta de afeto, atenção e carinho, que irão favorecer a segurança, o bem-estar e o alívio das sensações dolorosas do trabalho de parto. Esse apoio emocional deve ser estendido à família e ao acompanhante, que também ajudam no suporte durante esses momentos. ${ }^{17}$ A doula acolhe, cuida e conforta. ${ }^{18}$

Neste sentido, a maneira como a assistência é oferecida implica, diretamente, na satisfação de quem recebe, em especial, no acolhimento às mulheres no processo de parturição, no qual a satisfação, geralmente, se dá a partir das relações interpessoais. Portanto, um contexto favorável fortalece vínculos familiares essenciais para o desenvolvimento saudável do ser humano. ${ }^{17,19} \mathrm{Em}$ suma, possuir informações sobre o parto, ter controle sobre o evento, o grau de relaxamento, opiniões positivas sobre a equipe que prestou assistência, de forma cuidadosa e afetuosa, além da presença de um acompanhante, são percepções positivas. ${ }^{20}$

Por outro lado, as negativas são descritas pelas mulheres como solidão,
ISSN $2236-198$

sofrimento e abandono, algumas vezes associadas à ausência de um acompanhante, determinada por imposição institucional. ${ }^{17,21}$ Os autores $^{7,14}$ destacaram a ocorrência de situações em que houve ausência ou falta de orientação da equipe médica e de enfermagem, ocasionando malestar nas mulheres.

A má atenção da equipe, evidenciada pelo distanciamento dos profissionais em relação ao cuidado, tem sido demonstrada como um dos fatores geradores de insatisfação e incrementadores dos níveis de ansiedade das parturientes. ${ }^{21-22}$ Nesse sentido, são necessárias reflexões frente ao desrespeito e à desatenção dos profissionais às mulheres na condição de parturientes. ${ }^{20,23}$

O enfermeiro divide seu tempo, da jornada diária de trabalho, entre as ações gerenciais e assistenciais, mesmo quando está alocado para a atividade de assistência. Esta ocorrência corrobora na falta de acompanhamento profissional ${ }^{20}$ e assim, fragiliza a assistência. ${ }^{24} \mathrm{~A}$ função gerencial foi a que mais especificamente foi percebida pelos membros da equipe de saúde, principalmente, pelas relações e interconexões que se estabelecem em um sistema de cuidados. ${ }^{25}$ Precisa-se atentar para que as atividades gerenciais do cuidado não restrinjam as assistências.

As gestantes consideram que a acompanhante ajudou na provisão de recursos e a enfermagem reconhece que a presença da doula facilitou a realização das atividades administrativas, mas há evidências que muito precisa ser feito para que o 
papel da doula seja reconhecido e validado. ${ }^{14}$ Entretanto, o processo de inserção da doula, como uma experiência positiva, não foi compartilhado por todos os profissionais, gerando dificuldades inerentes às percepções da atuação da doula. ${ }^{7}$ Todavia, autores ${ }^{5,7}$ reconhecem a importância da doula no que se refere ao apoio emocional, substituição da família e prestação de orientação sobre a evolução do trabalho de parto. ${ }^{5,7}$

A recente e insipiente atuação das doulas nos serviços de saúde brasileira gera indagações, dificuldades de compreensão sobre os atributos das doulas e certa resistência da equipe de saúde quanto ao relacionamento profissional estabelecido entre esses sujeitos. ${ }^{5-26}$ Autores $^{7-8}$ enfatizam que além do desconhecimento pelos profissionais da saúde sobre as doulas, tem também a falta de informações e a compreensão necessária por parte das próprias parturientes e usuários do Sistema Único de Saúde (SUS) sobre as doulas. ${ }^{5,18,26}$

Destaca-se que há evidências qualitativas sobre o acompanhamento por doulas no trabalho de parto e no parto. $^{7}$ Neste sentido, há de considerar-se que os cuidados prestados pelas doulas empoderam e serenam as mulheres no momento do parto ${ }^{7}$, pois, o apoio vivenciado pelo acompanhante é positivo e benéfico para a mulher. Assim sendo, corroborase com o fato de que os profissionais podem e devem adaptar-se a essa nova realidade no momento do parto e, até mesmo, incentivar o processo de parturição com acompanhamento. 1,5,2728

\section{Práticas usadas no alívio da dor}

0 apoio emocional foi citado em cinco (45\%) dos artigos analisados $1,5,7,27,29$ como sendo a prática usada para promover o alívio da dor nas parturientes acompanhadas pelas doulas. As estratégias para tal foram conversar, dar informações para superar medos e anseios ${ }^{1,5,7}$ realizações de elogios e incentivos e contato visual para 0 empoderamento das parturientes. ${ }^{27}$

0 desconhecido é um dos fatores fundamentais que dificultam 0 trabalho de parto, amedronta as parturientes e seus familiares, que ficam excluídos deste processo. ${ }^{26} 0$ modo de cuidar utilizado pelas doulas rompe com o tecnicismo biomédico e, assim, proporcionam maior tranquilidade e naturalidade ao processo de parturição ao fornecerem orientações para aliviar a dor, informações sobre o recém-nascido, auxílio nos cuidados com o bebê $e$ amamentação, e predominantemente, promovem 0 apoio emocional. ${ }^{27}$ Portanto, o suporte intraparto oferecido pelas doulas é de suma importancia. ${ }^{30}$

A utilização de práticas benéficas recomendadas pela OMS para uma assistência adequada ao trabalho de parto, mudanças no modelo de atenção ao parto a fim de torná-lo uma experiência menos dolorosa e mais gratificante devem ser implementadas a curto prazo. ${ }^{31}$

As medidas de bem-estar e de conforto físico foram relatadas em 
dos

estudos, massagem $^{12,18,29}$ hidroterapia ${ }^{1,7,12,29}$ e deambulação ${ }^{12,18,30}$ foram as técnicas mais utilizadas no manejo da dor. Os exercícios respiratórios, mudança de posição ${ }^{12,29}$ musicoterapia ${ }^{13}$, suporte ao parceiro ${ }^{7,14}$, aromaterapia, áudioanalgesia e acumpultura ${ }^{14}$, também, foram especificadas como alternativas para o alívio da dor. Os estudos ${ }^{12,18,32-}$ 33 detalharam a eletroestimulação, o uso do cavalinho e da bola suíça como meios eficazes para manejar a dor no processo de parturição.

A atuação das doulas modifica as ações em saúde e transforma a qualidade de vida de mulheres submetidas ao seu acompanhamento. ${ }^{29}$ A utilização de métodos naturais para alívio da dor durante a gestação, o parto e 0 puerpério favorece 0 processo de parturição. ${ }^{34}$ Todavia, um fator a considerar-se é a pouca produção de estudos nacionais, no que se refere como são desenvolvidas as técnicas no momento da parturição ${ }^{31}$, com enfoque nos métodos não farmacológicos e não invasivos de alívio da dor.

\section{CONSIDERAÇÕES FINAIS}

A metodologia utilizada oportunizou expandir o conhecimento acerca da produção científica relacionada aos benefícios de acompanhante treinada durante 0 processo de parturição, permitindo um aprofundamento de informações sobre este assunto, maior instrumentalização frente a esta forma humanizada e qualificada de assistir a mulher durante o processo de parturição.

$\mathrm{Na}$ vivência das mulheres que tiveram acompanhamento por doula,
ISSN 2236 - 1987

os dados evidenciam satisfação das parturientes que receberam o apoio pela acompanhante. Por outro lado, em alguns casos, a falta de informação e comunicação com a equipe geraram insatisfação.

Das práticas usadas no alívio da dor, identificadas, tem-se o suporte emocional o qual envolve conversa com a parturiente, a fim de tranquilizá-la, favorecer o contato com os profissionais e com os familiares, além de ser uma presença amigável e constante para a parturiente e seus familiares. Evidenciou-se, também, que as medidas de bem-estar e conforto físico têm um efeito fundamental no processo de alívio da dor, as mais citadas, foram massagem, hidroterapia e deambulação.

A escassez de estudos relacionados à assistência prestada no momento da parturição, práticas usadas no alívio da dor e a vivência do acompanhamento por doula, ratificam a importância de se fazer novos estudos que abordem essa temática, e consequentemente, sejam criados subsídios capazes de possibilitar uma prática mais humanizada, efetiva e eficaz às parturientes.

Assim, recomenda-se um aprofundamento desta temática para que haja uma sensibilização coletiva sobre o assunto, compreensão da real importância desta discussão, enfocando, principalmente os benefícios de acompanhante treinada durante o processo de parturição. Espera-se que 0 acesso da acompanhante treinada (doula) às maternidades seja mais breve e assim, possa contribuir na humanização e na 


\section{NURSING \\ ANO HEALTH}

qualificação do processo de parturição.

Devido a atuação das doulas ser recente no país, as produções científicas são numericamente limitadas, todavia, proporcionam informações importantes acerca dessa nova ocupação de saúde e favorecem incremento ao debate acerca da humanização do ciclo gravídicopuerperal.

Para o aprimoramento dessa importante temática, sugere-se prosseguimento com novos estudos, incluindo trabalhos internacionais para ampliar as possibilidades interpretativas dos resultados acerca dos benefícios da acompanhante treinada durante $o$ processo de parturição.

\section{REFERÊNCIAS}

1. Leão MRC, Bastos MAR. Doulas apoiando mulheres durante o trabalho de parto: experiência do Hospital Sofia Feldman. Rev latino-am enfermagem. 2001 maio;9(3):90-4.

2. Organização Mundial da Saúde (OMS). Maternidade Segura. Assistência ao parto normal: um guia prático. Genebra; 1996.

3. Ministério da Saúde (BR). Secretaria de Atenção à Saúde. Departamento de Atenção Básica. Atenção ao pré-natal de baixo risco. Parto, aborto e puerpério: assistência humanizada à mulher. Brasília; 2001.

4. Brüggemann $O M$, Parpinelli $M A$, Osis MJD. Evidências sobre o suporte durante o trabalho de parto/parto: uma revisão da literatura. Cad saude publica. 2005;21(5):1316-27.
ISSN $2236-1987$

5. Santos DS, Nunes IM. Doulas na assistência ao parto: concepção de profissionais de enfermagem. Esc Anna Nery. 2009 set;13(3):582-8.

6. Ministério da Educação (BR). Instituto Federal de Educação, Ciência e Tecnologia de Brasília. Chamada Pública 005, 07 de dezembro de 2016: curso de extensão formação de doulas. Brasília; 2016.

7. Silva RM, Barros NF, Jorge HMF, Melo LPT, Ferreira Junior AR. Evidências qualitativas sobre o acompanhamento por doulas no trabalho de parto e no parto. Cienc saude colet. 2012 out;17(10):2783-4.

8. Ministério da Saúde (BR). Secretaria de Atenção à Saúde. Departamento de Atenção Básica. Atenção ao pré-natal de baixo risco. Brasília; 2012.

9. Diniz CSG. Humanização da assistência ao parto no Brasil: os muitos sentidos de um movimento. Cienc saude colet. 2005 set;10(3):62737.

10. Brasil. Portaria $n^{\circ}$ 569, de 01 de junho de 2000. Instituição do Programa de Humanização do Pré-natal e Nascimento no âmbito do Sistema Único de Saúde. Diário Oficial da República Federativa do Brasil. Diário Oficial da União. 8 jun 2000; Seção1: 110-E.

11. Leão VM, Oliveira SMJV. O papel da doula na assistência à parturiente. Reme, rev min enferm. 2006 $\mathrm{jan} / \mathrm{mar} ; 10(1): 24-9$.

12. Mafetoni RR, Shimo AKK. Métodos não farmacológicos para o alívio da dor no trabalho de parto: revisão integrativa. Reme, rev min enferm. 2014 abr/jun;18(2):505-12. 


\section{JOURNALOF \\ NURSING \\ ANO HEALTH \\ ISSN 2236 - 1987}

20];10(2):337-46. Disponível em: https://www.fen.ufg.br/fen_revista/ v10/n2/v10n2a05.htm

21. Velho MB, Santos EKA, Brüggemann OM, Camargo BV. Vivência do parto normal ou cesáreo: revisão integrativa sobre a percepção de mulheres. Texto contexto enferm. 2012 maio/ago;21(2):458-66.

22. Oweis A. Jordanian mother's report of their childbirth experience: findings from a questionnaire survey. Int $\mathrm{j}$ nurs pract. 2009 dez; 15(6):525-33.

23. Baston $H$, Rijnders $M$, Green $J M$, Buitendijk S. Looking back on birth three years later: factors associated with a negative appraisal in england and in the netherlands. J reprod infant psychol. 2008 nov;26(4):323-39.

24. Mendes EV. $O$ cuidado das condições crônicas na atenção primária à saúde: o imperativo da consolidação da estratégia da saúde da família. Brasília: Organização Pan-Americana da Saúde; 2012.

25. Backes DS, Backes MS, Erdmann AL. O papel do enfermeiro no contexto hospitalar: a visão de profissionais de saúde. Cienc cuid saude. 2008 $\mathrm{jul} / \mathrm{set}$;7(3):319-26.

26. Luz LDP. Inserção e atuação das doulas no sistema único de saúde: uma metassíntese [monografia]. Foz do Iguaçu (PR): Universidade Federal de Integração Latino-Americana; 2016.

27. Longo CSM, Andraus LMS, Barbosa MA. Participação do acompanhante na humanização do parto e sua relação com a equipe de saúde. Rev eletrônica enferm [Internet]. $2010 \mathrm{abr} /$ jun [acesso em $2016 \mathrm{dez} 20$ ];12(2):386-91. Disponível

em: maio/ago [acesso em 2016 dez 


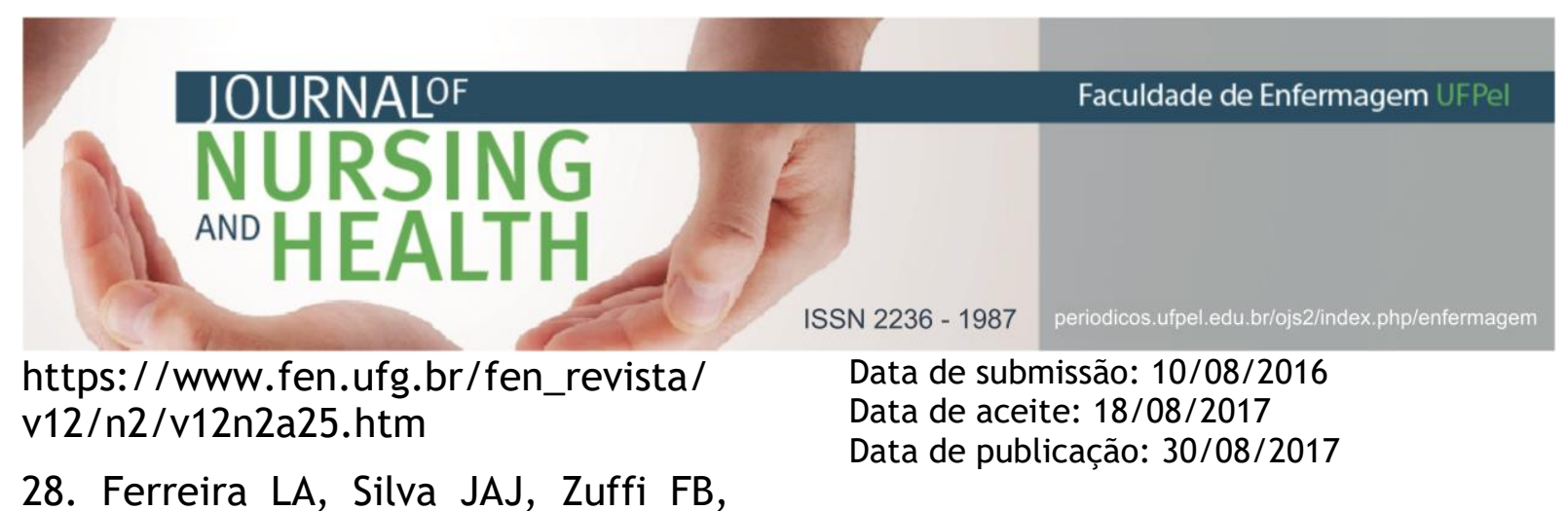
Mauzalto ACM, Leite CP, Nunes JS. Evidências qualitativas sobre 0 acompanhamento por doulas no trabalho de parto e no parto. Cienc saude colet. 2013 abr/jun;17(10):2783-94.

29. Lima MS, Moreira KA, Martins-Melo FR. A atuação da enfermagem na humanização do parto uma revisão integrativa. Revista tendências da enfermagem profissional. 2012 nov/dez 4(4):727-32.

30. Pitaluga LKSB. Qualidade de vida de mulheres submetidas ao acompanhamento de doulas [dissertação]. Goiânia (GO): Pontifícia Universidade Católica de Goiás; 2014.

31. Domingues RMSM, Dias MAB, Nakamura-Pereira M, Torres JA, Orsi E, Pereira APE, et al. Processo de decisão pelo tipo de parto no Brasil: da preferência inicial das mulheres à via de parto final. Cad saude publica. 2014 out/nov;30 Suppl:S101-16.

32. Gallo RBS, Santana LS, Marcolin AC, Quintana SM. A bola suíça no alívio da dor de primigestas na fase ativa do trabalho de parto. Rev dor. 2014 dez;15(4):253-5.

33. Crizóstomo CD, Nery IS, Luz MHB. A vivência de mulheres no parto domiciliar e hospitalar. Esc Anna Nery. 2007 mar;11(1):98-104.

34. Souza SV, Scheid AO. Percepções de doulas naturólogas sobre gestação, parto e puerpério. Cadernos de naturologia e terapias complementares. 2014 jul/set;3(4):4353. 